\title{
On the relative frequency of depth effects in real versus illusory figures
}

\author{
THEODORE E. PARKS \\ University of California, Davis, California
}

\begin{abstract}
Illusory depth in an outlined square and in an illusory square was assessed by requiring subjects to choose among phenomenological descriptions and to report the relative sizes of dots located inside and outside these squares. Contrary to previous suggestions, results obtained with the two measures were not mutually contradictory regarding depth and neither measure suggested that depth effects are less frequent in illusory figures.
\end{abstract}

A number of recent studies have compared illusory figures to physically complete ("real") figures on several dimensions. For example, researchers have compared the respective abilities of these figures to produce geometric illusions (see review in Parks, 1984), to resist fragmentation under fixed-image conditions (Halpern \& Warm, 1980), and to induce an illusory-lightness effect (e.g., Parks, Rock, \& Anson, 1983). In the same vein, Porac (1978) attempted to compare the tendency to see illusory depth in an illusory-figure pattern (similar to Figure 1a) with the same tendency in the same pattern when the area of the figure is outlined (as in Figure 1b).

Unfortunately, the results of this last endeavor were somewhat contradictory. To be specific, in one part of her research, Porac (1978) asked her subjects to choose a description of their experience of each of the two patterns from among a set of potential descriptions. She found that a descriptive phrase that included the idea that the square was "in front" was chosen more often by subjects viewing a real square than by subjects viewing an illusory square. By contrast, however, she found that the illusory figure was superior when apparent depth was assessed by a second measure: the tendency for a dot placed within either figure to appear to be smaller than an identical dot placed to the outside (see Figures $1 \mathrm{c}$ and 1d) an effect that supposedly resulted from inappropriate size scaling in response to an illusory-depth difference. Specifically, responses to this measure suggested the presence of a depth effect only in the illusory-square display and not in the real display.

Various explanations are possible for this discrepancy between the results Porac (1978) obtained using one of her measures as compared with the other. For example, the subjects who chose among the verbal descriptions of depth were not the same as those who were presented with the two-dot displays and were asked about relative dot sizes. Also, a careful review of the precise wording of

The author is indebted to Ryung Kim and Lisa Curtis for their excellent assistance in collecting the present data. Reprint requests should be addressed to the author at the Department of Psychology, University of California, Davis, CA 95616. the descriptions Porac offered to the former group suggests that she might have forced those subjects to choose the in-front alternative for the real-square display even if no depth was actually experienced. That is, the alternatives she supplied read: (1) "four black circles connected by lines" and (2) "four black circles with a wedge cut out of each one" for the two no-depth alternatives, and (3) "a white square in front of four black circles" for the single depth-present alternative. The first of these choices was somewhat inaccurate if depth was not experienced, because it implied whole circles-an inappropriate description unless the square seemed to be occluding them (which, without depth, would not be the case). The second description is even more inadequate for the real display because it doesn't even mention the lines that are physically there. Finally, and more importantly, only the third alternative (the depth-present choice) mentions the salient fact that those lines form a square.

Nevertheless, any subject who experienced no depth in the illusory-square pattern was provided with a clean nodepth choice in the second alternative listed above. An alternative explanation, then, for the apparent superiority of the real figure in eliciting choices of a depth-present description could be that it was an artifactual product of wording.

Consequently, the aim of the first of the present experiments was to establish more firmly the generality of Porac's (1978) intriguing findings, using a more uniformly worded set of alternative verbal descriptions. At the same time, the effects Porac examined were reexamined using the size-scaling technique, but this time with the same subjects who also chose among verbal descriptions.

\section{EXPERIMENT 1}

\section{Method}

Subjects and Materials. A total of 96 undergraduate volunteers were individually shown the four patterns of Figure 1 (from a distance of approximately $2 \mathrm{~m}$ and under ordinary room illumination) after being given general instructions that emphasized phenomenological accuracy and stated that there were "no correct responses, just what is true for you." 

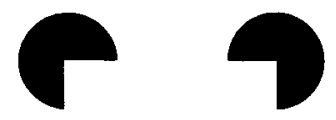

a
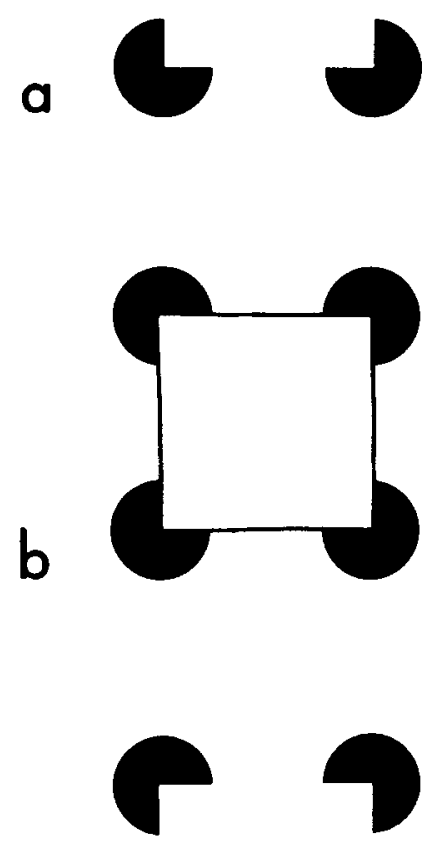

.

c
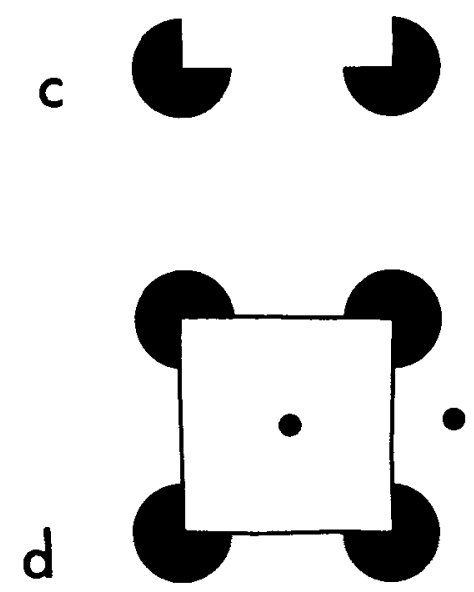

Figure 1. The patterns (each approximately $7 \mathrm{~cm}$ in overall height) used in Experiment 1.

Procedure and Design. The subjects were first shown a rather powerful inducing pattern consisting of Pattern la with the additional inclusion of a black half-circle lying along, and centered on, each edge of the illusory square. The pattern was presented in the belief that, given the current popularity of illusory figures, at least some of these volunteers would have seen one of them prior to their participation in the present experiment. Its presentation ensured that they all would have had such experience prior to the trials of interest here. At this time, each subject was simply asked, "Do you seem to see a white square in [the] pattern?' All replied affirmatively.
Each subject was then shown Patterns 1c and 1d, one at a time and in balanced order across subjects, and in each case was asked, "If you had to choose, which of these two dots seems to be the bigger?" For any given subject, the outside dot was always to the left or to the right; this arrangement also was balanced across subjects and across order of presentation.

Following these two trials, the subjects were shown Patterns 1a and $1 \mathrm{~b}$. As before, the patterns were shown separately and in balanced order across subjects and across the two variables (order and dot-position) of the first two trials. Upon viewing each of these patterns, each subject was handed one of two cards, on each of which the following two statements appeared: "This looks like a white square connecting pieces of four black circles" and "This looks like a white square in front of four black circles." The two cards differed in the order in which the two descriptions appeared and were used equally often across the values of the other variables.

\section{Results and Discussion}

Even with the improved wording of the descriptions that were offered here-specifically, having the no-depth alternative mention the "square" and "pieces of circles" -as in Porac (1978), by far the most popular response to the outlined pattern (Pattern 1b) was the depth alternative, which was chosen by 81 of the 96 subjects. In further confirmation of Porac's results, the depth-alternative choice for the illusory-figure pattern was not as popular; this choice was made by only 67 of the 96 subjects, in a ratio that was very close to the approximately $2: 1$ ratio she obtained. For this difference between patterns, $\chi^{2}(1)$ $=4.225, p<.05$. The important implication is that the superiority of a real figure over an illusory one by this measure was not an artifactual product of wording.

However, the remainder of the present results did not strictly support Porac's (1978) other finding, which was that the relative strengths of the two displays reverse when depth is evaluated by the size-scaling technique. To be sure, 85 of 96 of the present subjects did indicate that the outside dot in the illusory-square pattern appeared to be the larger of the two (thus suggesting that to a strong majority the inside dot-and, therefore, the square upon which it was located-appeared to be nearer). However, 86 of these subjects made the same response to the realfigure pattern, so the relative strengths of the two displays did not literally reverse in the present case.

On the other hand, an essential aspect of the puzzle inherent in Porac's (1978) data remains even in these results. That is, by one measure the real-square pattern is clearly superior, whereas by the other it is not (and, in this case, even though the same subjects participated in both procedures).

\section{EXPERIMENT 2}

Following Experiment 1 certain questions remained, particularly with respect to the illusory-figure results. Specifically, it is curious that, if the depth effect produced by Pattern 1c was strong enough to induce a noticeable size-scaling effect in 85 subjects, this depth effect was reported by only 67 of these subjects in response to a direct query in the other condition (Pattern 1a). 
One attractive possibility is that a depth effect did occur in only about 67 of the observers in each measurement condition, and that in the size-scaling condition the remaining 29 subjects were forced to choose randomly between the two dots. If so, the expected result [67+ $.5(29) \cong 82$ subjects] would approach the frequency that was actually obtained for these trials (i.e., 85 subjects). However, one difficulty with this explanation is that it ignores the fact that a very similar size-scaling result was obtained for both squares. By contrast, according to this proposal, because the real square was clearly superior with respect to description choices, it should also be clearly superior with respect to size scaling (the latter supposedly consisting of the former frequency plus "correct" guesses), but it was not.

A second possible explanation for the illusory-figure results invokes the widely proposed principle that illusorydepth differences that contribute to inappropriate size scaling in various illusions are not always available to introspection (e.g., Gregory, 1966; Rock, 1975), so that the fact that the frequency of the size-scaling effect was greater than the frequency of depth descriptions is not surprising: the former overestimates the true frequency of a visible depth effect.

This explanation is also troublesome, however, because it ignores the fact that very similar size-scaling results were obtained for both patterns. That being the case, the second proposal would predict that the description-choice data should also be similar (because both frequencies were expected to be lower than the size-scaling result and, particularly, lower by a similar amount), but they were not.

A review of the geometric-illusion literature suggests a third, potentially better, explanation for the relatively high frequency of the size-scaling effect, albeit one that might drastically alter the effect's explanation, as well as that of related experiments (i.e., Coren, 1972; Parks, 1985). That is, Ebbinghaus (1902; see Rock, 1975) found that a small circle looks smaller when surrounded by larger circles than when surrounded by smaller circles. Clearly, the former condition is very similar to the critical parts of the size-scaling displays (Patterns $1 \mathrm{c}$ and 1d) with respect to the inside dots, the only variation being that the present surrounding circles are not complete. On the other hand, an extreme version of the latter condition, in which the surrounding circles were so small as to be undetectable, would mimic the situation of the present outside dots to at least one of their sides. The suggestion, then, is that the dot-size effect might occur in displays such as the present one even when all clues to occlusion, and therefore to depth, are eliminated. Experiment 2 tested this possibility.

In addition, further evidence was sought to support the results obtained for Experiment 1 and, specifically, to explore an additional possible explanation for the relatively low proportion of subjects who chose a depth description for the illusory figure. Previous attempts to assess illusoryfigure depth have shown that depth occasionally occurs in the direction opposite to that of the usual effect; the area of the usual illusory figure appears, instead, as an opening or "window" through which a recessed background is viewed (Halpern, 1981; see Parks \& Marks, 1985 , for the same point, but with respect to a different pattern). Since none of the phenomenological descriptions provided by Porac (1978) or reported here included this possibility, any such occurrences would be expected to have resulted in reports of no depth (i.e., subjects would reasonably have preferred such a choice over the even more contradictory idea that the square appeared to be in front of the black inducing elements). Thus, this aspect of the previous results might simply reflect a tendency of the illusory-figure pattern sometimes to produce an alternative arrangement in apparent depth-a tendency that might not be shared equally by the outlined version. If so, relatively more subjects might have experienced depth in the illusory pattern than the description-choice results seem to indicate. In an attempt to evaluate this possibility, such window descriptions were included among the set of phenomenological alternatives offered to the subjects in this portion of Experiment 2.

\section{Method}

Subjects, Materials, and Procedure. A new group of 48 subjects were treated identically to those of Experiment 1 through the point at which the practice illusory-figure pattern was presented. Following that, they were shown a modified version of Pattern 1c in which the circles were complete (see Figure 2) and queried as in Experiment 1. Once again, the outside dot was equally often located to the left and to the right.

Each subject was then shown Pattern la and was handed three cards; two of these each contained one of the two descriptive sentences employed in Experiment 1 (except that the word "white" was omitted from both) and the third said, "This looks like a square opening between pieces of four black circles." The word "white" was avoided because of uncertainty as to whether the usual lightness effect would occur as reliably with the window depth effect as with the usual in-front result. If it did not, this third choice might sometimes have been less attractive because of the word "white." At any rate, the lightness effect is not at issue here.

The order in which the three cards were stacked was predetermined, each of the six possible orders being used equally often across subjects.

\section{Results and Discussion}

Despite the absence of depth cues to suggest that the outside dot was farther than the other dot, 39 of $48 \mathrm{ob}$ -

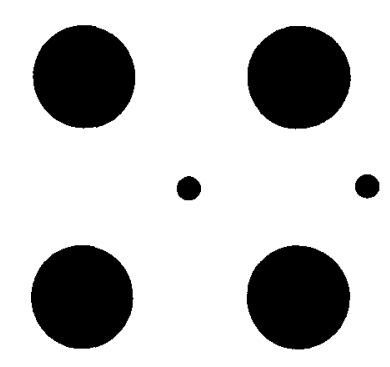

Figure 2. The modified (nonocclusion) pattern used in Experiment 2. 
servers $(81 \%)$ reported that this dot appeared to be larger, a frequency that is quite close to the overall results of Experiment 1 (in which $89 \%$ of the subjects so chose).

Apparently, then, in some occlusion patterns (in particular, in the patterns used in Experiment 1) the size effect cannot be attributed with any certainty to a sizescaling effect based upon illusory depth. This is not to say that no such depth occurred, but only that the Ebbinghaus (1902) effect would have produced the size effect in most subjects even in the absence of any depth effect. ${ }^{1}$ That the size effect occurred more frequently or that no difference was found between patterns by this measure is therefore no longer surprising. The more general point is that the mere occurrence of a size effect in an illusoryfigure pattern can no longer be taken as conclusive evidence for illusory depth.

However, because in other cases differences in the frequency of the size effect are found between patterns even when those patterns seem to be identical with respect to the Ebbinghaus effect, the Ebbinghaus effect may be so weak in them as to allow illusory depth to predominate. This may have been the case in the patterns used by Porac (1978). (Note, for instance, that her circle elements were proportionately smaller than those of the present experiment.)

If so, however, a conflict remains within Porac's (1978) results because depth was reported relatively less frequently in the real figure by one measure and relatively more frequently by the other. The resolution of this conflict in Porac's data may, however, lie in an additional fact: illusory depth, when it occurs, has been found to be of greater magnitude in illusory figures than in real ones (Coren \& Porac, 1983). Accordingly, even though an in-front depth arrangement was observed by fewer of her subjects in the illusory pattern than in the real one, this effect may have been of great enough magnitude to also produce a detectable size effect. Conversely, although most subjects may have experienced depth in her real figure, very few, if any, of them may have experienced a depth effect of sufficient magnitude for an apparent effect on dot size to be produced. In this sense, then, illusory figures might actually be considered to be superior to real ones despite the relatively low frequency of depth descriptions they elicit.

Furthermore, it is essential to remember that even this relatively low frequency concerns only the "in-front" arrangement in depth. As noted earlier, some failures to achieve this arrangement might, nevertheless, reflect a depth effect inasmuch as an alternative depth arrangement (the window effect) might have occurred instead. In fact, the verbal-description choices made in Experiment 2 satisfy this expectation dramatically: of the 8 subjects who did not choose the "in-front" statement, 5 chose the square-opening option instead. Thus, only 3 of 48 subjects reported no depth when a description of either possible depth arrangement was allowed.

Strikingly, this overall frequency of depth reports (45 of 48 , or $94 \%$ ) exceeds even that obtained for the real figure in Experiment 1 ( 81 of 96 , or $84 \%$ ). Although this result might be somewhat atypical-Halpern (1981) obtained relatively fewer reports of reverse depth and relatively more of no depth-it leaves little room for serious concern about the ability of illusory-figure patterns to induce illusory depth of either form, and to do so, in some cases, as frequently as does a real-figure pattern.

\section{REFERENCES}

Coren, S. (1972). Subjective contours and apparent depth. Psychological Review, 79, 359-367.

CoRen, S., Porac, C. (1983). Subjective contours and apparent depth: A direct test. Perception \& Psychophysics, 33, 197-200.

Ebbinghaus, H. (1902). Grundzuge der Psychologie. Leipzig: von Veit. GREGORY, R. L. (1966). Comments on Dr. Vernon Hamilton's paper. Quarterly Journal of Experimental Psychology, 18, 73-74.

HaLPERN, D. F.(1981). The determinants of illusory-contour perception. Perception, 10, 199-213.

HALPERN, D. F., WARM, J. S. (1980). The disappearance of real and subjective contours. Perception and Psychophysics, 28, 229-235.

PARKs, T. E. (1984). Illusory figures: A (mostly) atheoretical review. Psychological Bulletin, 95, 282-300.

Parks, T. E. (1985). Apparent depth and texture differences in illusory figure patterns: A paradox resolved. Perception \& Psychophysics, 37, 568-570.

Parks, T. E., \& Marks, W. (1985). Mlusory figures: Individual differences in apparent depth and lightness. Perception \& Psychophysics, 37, 529-532.

PARKs, T. E., Rock, I., a Anson, R. (1983). Illusory contour lightness: A neglected possibility. Perception, 12, 43-47.

PorAc, C. (1978). Depth in objective and subjective contour patterns. Bulletin of the Psychonomic Society, 11, 103-105.

Rock, I. An introduction to perception. New York: Macmillan.

\section{NOTE}

1. The same could be said when many dots are present, as when the inducing elements are presented on a field of small dots to study their effects on apparent texture. This principle might even account for the finding that black dots within an illusory figure look smaller than outside dots only when the inducing elements are themselves black (Parks, 1985, Experiment 1 vs. Experiment 3).

(Manuscript received August 6, 1986; revision accepted for publication March 16, 1987.) 